\title{
LA TUTELA PENALE DEI BENI CULTURALI DAL TRAFFICO ILLECITO, DAL DANNEGGIAMENTO E DA ALTRE FORME DI AGGRESSIONE ALLA LUCE DEL DISEGNO DI LEGGE ITALIANO AC- 4220
}

\author{
THE CRIMINAL PROTECTION OF CULTURAL ASSETS FROM \\ ILLECIT TRAFFICKING, DAMAGE AND OTHER FORM OF \\ AGGRESSION IN THE LIGHT OF THE ITALIAN BILL AC- 4220
}

ALICE CAPUTO*

\begin{abstract}
Il presente lavoro analizza l'attuale tutela penale del patrimonio culturale nell'ordinamento italiano e il recente disegno di legge AC 4220, recante disposizioni in materia di reati contro il patrimonio culturale, proposto proprio al fine di rafforzarne la tutela. L'esteso patrimonio artistico, culturale ed archeologico del nostro Paese richiede, infatti, una tutela di tali beni, dal traffico illecito, dal depauperamento, dal danneggiamento e da altri reati collegati, sempre più effettiva ed efficace. Di fatto, si tratta di beni comuni, di interesse pubblico generale ed intergenerazionale che permettono all'autore/ artista di trasmettere nel tempo il seme della sua creatività e di trasformarlo in una rosa, dalla bellezza variegata a seconda dello sguardo di chi la coglie, nella contemplazione da parte del pubblico.
\end{abstract}

KEYWORDS: Beni Culturali. Traffico Illecito. Tutela Penale. Riforma Codice Penale.

\begin{abstract}
The present work analyzes the current criminal protection of cutlural heritage in the italian legal system and the recent draft law AC 4220 , containing provisions on crimens against cultural heritage, proposed precisely in order to strengthen ist protection. The extensive artistic, cultural and archaeological heritage of our country requires, in fact, a protection of theseassets, from illicit traffic, depletion, damage and other related crimes, more and more effective and effective. In fact, these are common goods, of general and intergenerational public interest that allow the authorlartist to pass on the seed of his creativity over time and trasform it into a rose, with a variegated beauty depending on the gaze of those who see it, in contemplation by the public.
\end{abstract}

KEYWORDS: Cultural Heritage. Illecit Trafficking. Criminal Protection. Penal Code Reforme.

Sommario: 1. Premessa: il crescente rilievo del traffico illecito dei beni culturali. - 2. La tutela penale attuale del beni culturali prevista dal Codice dei Beni Culturali e del Paesaggio e dall'art. 733 c.p. 3. Il recente disegno di legge AC 4220: l'assorbimento nel Codice penale delle fattispecie penali a tutela del patrimonio culturale. - 4 . Qualche osservazione conclusiva.

Contract professor of criminal law at the SSPL of the second university of Naples. Lawyer.

E-mail: alicecaputo@virgilio.it. 


\section{PREMESSA: IL CRESCENTE RILIEVO DEL TRAFFICO ILLECITO DEI BENI CULTURALI}

Il traffico illecito di beni culturali, artistici e archeologici ha assunto un tale rilievo, negli ultimi anni, da rendere sempre più urgente una legislazione penale di contrasto sia a livello interno che internazionale ${ }^{1}$. Tale rilievo ha reso il traffico illecito di beni culturali, realizzato tramite i furti di opere, i saccheggi dei siti archeologici, il traffico di beni durante i conflitti armati e per il finanziamento del terrorismo, uno dei mercati illegali più redditizi, oltre a quelli già ben noti della droga e delle armi. Cosicché il Consiglio d'Europa, a maggio 2017, ha approvato il primo trattato internazionale dedicato all'incriminazione del traffico illecito dei beni culturali, assicurando così un'efficace azione penale in chiave preventiva e repressiva. In particolare, il Consiglio d'Europa punta a salvaguardare il patrimonio mondiale spingendo gli Stati a punire con sanzioni effettive i trafficanti di beni culturali e coloro che li distruggono, passando attraverso il diritto penale ${ }^{2}$. La Convenzione punisce, in particolare, i furti, gli scavi illegali, l'importazione e l'esportazione illecita, l'acquisizione e la commercializzazione di questi beni, il danneggiamento internazionale dei beni ed invita gli Stati a predisporre sanzioni effettive, proporzionali e dissuasive nei confronti di persone fisiche e giuridiche. Così come prevede circostanze aggravanti, se l'infrazione è commessa nel quadro di un'organizzazione criminale o di recidiva, così come nei casi in cui il reato sia commesso da funzionari responsabili dei beni culturali. L'Italia, che con il suo ricco e straordinario patrimonio artistico e culturale, è uno dei Paesi maggiormente interessati da questo fenomeno, ha provveduto alla ratifica di tale trattato e attualmente, con il disegno di legge AC 4220, recante disposizioni in materia di reati contro il patrimonio culturale, potrebbe a breve

1 Il 19 maggio a Nicosia è stata adottata la Convenzione del Consiglio d'Europa sui reati relativi ai beni culturali: cfr: Council of Europe Convention on Offences relating to Cultural Property https://search.coe.int/cm/Pages/result_details.aspx?OObjectId=0900001680704b3. Dal Transnational Crime and the Developing World Report 2017 del Global Financing Integrity è emerso che il traffico illecito nel mondo dell'arte ha un valore annuo tra 6 e 8 miliardi di dollari, con ricavi annui pari a 1.2/1.6 miliardi di dollari, e rappresenta il $20 \%$ del complesso dei crimini contro il patrimonio culturale, l' $80 \%$ del quale, infatti, è rappresentato dalla contraffazione delle opere e dalla realizzazione ed uso di documenti falsificati. Secondo uno studio dell'associazione americana Save Antiquities for everybody, l'80\% dei beni venduti nei mercati d'arte ha origini incerte, se non illegali e, secondo l'Interpol, il traffico di beni culturali sarebbe secondo solo al commercio di armi e droga.

2 In dottrina, DONINI M., Il principio di offensività. Dalla penalistica italiana ai programmi europei, in Rivista Penale Contemporaneo; SOTIS C., Obblighi comunitari di tutela e opzione penale: una dialettica perpetua?, in Riv.it.dir. e proc.pen., 2002, pag. 171. PAONESSA C., Gli obblighi di tutela penale. La discrezionalità legislativa nella cornice dei vincoli costituzionali e comunitari, ETS, Pisa, 2009; VIGANÒ F., L'arbitrio del non punire. Sugli obblighi di tutela penale dei diritti fondamentali, in Studi in onore di M. Romano, Jovene, Napoli, 2011, vol., II, 2645. VAGLIASINDI G.M., Obblighi di penalizzazione di fonte europea e principi di politica criminale: le indicazioni promananti dalla materia ambientale, in L'evoluzione del diritto penale nei settori d'interesse europeo alla luce del Trattato di Lisbona, a cura di G. Grasso, L. Picotti, R. Sicurella, Giuffrè, Milano, 2011. 
dotarsi di una nuova riforma penale sul tema che prevede l'inserimento di un nuovo titolo del Codice Penale con nuovi reati e un rilevante inasprimento sanzionatorio. Il disegno di legge in questione pone interessanti profili di novità nel contrasto al traffico illecito di beni culturali, creando profili di avanguardia nella tutela penale del fenomeno. Nel presente lavoro, senza alcuna pretesa di esaustività, si cercheranno di tratteggiare i profili, de iure condendo, più rilevanti di tali novità, tenendo conto anche dell'attuale tutela prevista dal Codice dei Beni Culturali e del Paesaggio (decreto legislativo n. 42/2004).

\section{LA TUTELA PENALE ATTUALE DEL BENI CULTURALI PREVIS- TA DAL CODICE DEI BENI CULTURALI E DEL PAESAGGIO E DALL`ART. 733 C.P.}

Nell'ordinamento italiano, la tutela dei beni culturali è un principio fondamentale di rango costituzionale. Infatti, la Costituzione all'art. 9 sancisce che: „La Repubblica promuove lo sviluppo della cultura e la ricerca scientifica e tecnica.

Tutela il paessaggio e il patrimonio storico e artistico della Nazione“.

In quanto principio fondamentale, la tutela del patrimonio culturale permette al legislatore di inserire strumenti di prevenzione e repressione dei comportamenti lesivi del patrimonio culturale, artistico ed archeologico di natura penale, così come permette di considerare la tutela dei beni culturali come bene giuridico da tutelare ${ }^{3}$. La criminalizzazione del traffico e del depauperamento del traffico illecito di beni culturali sta acquisendo, negli ultimi anni, una rilevanza crescente.

Attualmente, è proprio il Codice dei Beni Culturali e del Paesaggio d.lgs. n. 42/2004, il quale ha abrogato il Testo Unico in materia di beni culturali e ambientali previsto dal d.lgs. n. 490/99 e ha sostituito le residue fattispecie previste dalla legge n. 1089/39, a costituire il punto di riferimento della tutela, anche penale, del patrimonio culturale. L'oggetto di tutela del Codice in questione riguarda i beni di rilievo culturale pubblici e privati, con una differenziazione nella definizione del rilievo culturale a seconda che essi siano pubblici o privati. Per i beni pubblici, secondo quanto previsto dell'art. 12, comma 1, del Codice dei Beni Culturali e del Paesaggio vige una presunzione di interesse storico ed artistico.

Infatti, secondo quando previsto dal suddetto art. 12 , sono da considerarsi

3 Per un'analisi approfondita cfr., SANDULLI A. M., La tutela del paesaggio nella Costituzione, in Riv. giur. edil., 1967, II, pag. 170. PREDIERI A., Significato della norma costituzionale sulla tutela del paesaggio, Le libertà civili e politiche, in Studi per il XX Anniversario dell'Assemblea Costituente, Vallecchi, Firenze, 1969, II, pag. 381. MERUSI F., Commento all'art. 9 Cost., in Commentario della Costituzione, a cura di G. Branca, Bologna, 1975, I, pag. 441. PELILLO A.R., I beni culturali nella giurisprudenza costituzionale: definizioni, poteri, disciplina, in Aedon, 2, 1998 pag. 35. MARINI F.S., Lo statuto costituzionale dei beni culturali, Giuffrè, Milano, 2002. In giurisprudenza, cfr. Corte Cost., Sent. n. 151 del 24 giugno 1986, in www. cortecostituzionale.it. 
beni culturali le cose mobili o immobili appartenenti allo Stato, alle Regioni, agli altri Enti pubblici territoriali, nonché ad ogni altro Ente o istituto pubblico e a persone giuridiche private senza fine di lucro, ivi compresi gli enti ecclesiastici, che presentino un semplice "interesse storico, artistico, archeologico o etnoantropologico". Rientrano nel raggio di tutela del Codice secondo quanto previsto dall'art. 10 , comma 1 , i beni mobili o immobili che costituiscano opere di autore non più vivente e la cui esecuzione risalga ad oltre cinquanta anni se mobile o ad oltre settanta anni se immobile 4 . Tali beni pubblici, così come appena delineati, godono di una tutela di presunzione, in pratica se tali beni rientrano nelle tipologie appena descritte il loro rilievo culturale, che li rende oggetto di tutela, viene presunto. La presunzione del valore culturale dei suddetti beni, secondo quanto stabilito dall'art. 12, comma 2 del Codice, sussiste, però, fino a quando non sia stata effettuata una verifica da parte del Ministero, che può avvenire d'ufficio o su istanza dei soggetti a cui le cose appartengono, circa la effettiva sussistenza dell'interesse culturale del bene. Infatti, se, all'esito di tale verifica sul bene non dovesse essere riscontrato alcun interesse culturale, lo stesso non godrà più delle norme di protezione contenute nel Codice dei Beni Culturali e del Paesaggio, con esclusione di alcuni beni tassativamente indicati dall'art. 10 comma 2, come ad esempio, le raccolte di musei, pinacoteche, gallerie, archivi, raccolte librarie delle biblioteche appartenenti allo Stato, alle Regioni, agli altri enti pubblici territoriali nonché ad ogni altro ente o istituto pubblico ${ }^{5}$.

Per i beni culturali di natura privata, la tutela penale è prevista solo in presenza del rilievo culturale derivante dalla dichiarazione di interesse culturale prevista dall'art. 13 del Codice debitamente rilasciata dalle competenti autorità, che ne attesti espressamente il valore storico, artistico e archeologico. In questi casi, a differenza dei beni culturali pubblici non sussiste nessuna forma di presunzione dell'interesse storico, artistico, archeologico o etnoantropologico, ma è necessaria una dichiarazione di rilievo rilasciata secondo le modalità previste dall'art. 14 del Codice stesso.

Definito l'oggetto della tutela penale secondo quanto previsto dal Codice, è possibile esaminare le ipotesi di reato previste dal Codice dei Beni Culturali e del Paesaggio. I reati previsti si distinguono in contravvenzioni e delitti. L'art. 169 lettera a) del Codice prevede la fattispecie di reato contravvenzionale punita con l'arresto da 6 mesi a 1 anno e con l'ammenda da euro 775 a euro 38.734,50 per chiunque, senza autorizzazione, demolisce, rimuove, modifica, restaura ovvero esegue opere di qualunque genere sui beni culturali indicati nell'art. 10. L'art. 169

4 AA. VV., Commentario al Codice dei beni culturali e del paesaggio, a cura di A. Angiuli V. Caputi Jambrenghi, Giappichelli, Torino, 2005. TAMIOZZO R., La legislazione dei beni culturali e paesaggistici, Giuffrè, Milano, 2005. MOCCIA S., Riflessioni sulla tutela penale dei beni culturali, in Riv.it.dir.e e proc.pen., 1993, p. 1294.

5 Sul punto, cfr. GIANNINI M.S., I beni culturali, in Riv. Trim. Di dir. Pubb., 1976, p. 4. 
lettera b) invece punisce: „Chiunque senza l'autorizzazione del soprintendente, procede al distacco di affreschi, stemmi, graffiti, lapidi, iscrizioni, tabernacoli ed altri ornamenti di edifici, esposti o no alla pubblica vista, anche se non vi sia stata la dichiarazione prevista dall'art. 13 ".

La pena prevista è uguale a quella prevista per il reato di cui all'art. 169 lett. a) del Codice.

La lettera c) dell'art. 169 punisce: „Chiunque esegue, in casi di assoluta urgenza, lavori provvisori indispensabili per evitare danni notevoli ai beni indicati nell'art. 10, senza darne immediata comunicazione alla soprintendenza ovvero senza inviare, nel più breve tempo, i progetti di lavoro definitivi per l'autorizzazione".

L'art. 170 del Codice stabilisce, invece, che: " $E$ 'punito con l'arresto da sei mesi ad un anno e con l'ammenda da euro 775 a euro 38.734,50 chiunque destina $i$ beni culturali indicati nell'art. 10 ad un uso incompatibile con il loro carattere storico od artistico o pregiudizievole per la loro conservazione o integrità".

L'art. 173 del Codice dei Beni Culturali e del Paesaggio delinea il delitto di alienazione senza autorizzazione ed altri reati ad esso collegati per i quali è prevista la sanzione della reclusione fino ad un anno e la multa da euro 1.549,50 a euro 77.469,00. Le condotte penalmente rilevanti sono la vendita senza autorizzazione, l'omessa denuncia dell'atto di trasferimento e la consegna della cosa in pendenza del termine relativo al diritto di prelazione.

L'art. 173 lett. a) punisce chiunque, senza la prescritta autorizzazione, aliena $i$ beni culturali indicati negli articoli 55 e 56".

L'art. 173 lett. b) chiunque, essendovi tenuto, non presenta al soprintendente del luogo dove si trovano i beni, nel termine di 30 giorni previsto dall'art. 59, comma 2 del Codice la denuncia degli atti di trasferimento della proprietà o della detenzione dei beni culturali.

L'art. 173 lett. c) del Codice, infine, punisce la condotta dell'alienante di un bene culturale soggetto a prelazione che effettua la vendita in pendenza del termine previsto dall'art. 61, comma 1, del Codice stesso, ossia nel termine di 60 giorni dalla data di ricezione della denuncia prescritta dall'art. 59.

All'art. 174 il Codice dei Beni Culturali e del Paesaggio punisce con la reclusione da 1 a 4 anni o con la multa da $€ 258,00$ a $€ 5.165,00$, chiunque trasferisca all'estero cose di interesse artistico, storico, archeologico, etnoantropologico, bibliografico, documentale o archivistico, nonché le cose oggetto di specifica tutela da parte dell'art. 11, comma 1, lett. f), g), h) (ovvero le fotografie gli esemplari di opere cinematografiche che hanno più di venticinque anni, i mezzi di trasporto aventi più di settantacinque anni, i beni e gli strumenti aventi interesse per la storia della scienza e della tecnica aventi più di cinquanta anni) senza attestato di libera circolazione o licenza di esportazione. Alla stessa pena soggiace anche il soggetto che non fa rientrare nel territorio nazionale, 
alla scadenza del termine stabilito, beni culturali per i quali sia stata autorizzata l'uscita o l'esportazione temporanee.

In riferimento a tali beni culturali, è possibile disporre la misura della confisca.

L'art. 175 prevede due distinte fattispecie di reato contravvenzionale entrambe punite con la pena dell'arresto fino ad un anno e l'ammenda da euro 310 a euro $3.099,00$.

La prima fattispecie riguarda: „Chiunque esegue ricerche archeologiche $o$, in genere, opere per il ritrovamento di cose indicate dall'articolo 10 senza concessione, ovvero non osserva le prescrizioni date dall'amministrazione".

La seconda fattispecie, invece, prevede due distinti reati di tipo omissivo. La prima condotta penalmente rilevante consiste nell'omessa denuncia, nel termine indicato dall'art. 90, comma 1 del D.Lgs. n. 42/04, dei beni culturali di cui all'art. 10 della stessa normativa rinvenuti fortuitamente. La seconda condotta riguarda l'inosservanza di provvedere alla conservazione temporanea delle cose oggetto di scoperta fortuita.

Infine, l'art. 176 del Codice tutela chiaramente il ritorno dei beni culturali nella disponibilità materiale e giuridica dello Stato. Cosicchè, "Chiunque si impossessa di beni culturali indicati nell'articolo 10 appartenenti allo Stato ai sensi dell'articolo 91 è punito con la reclusione fino a tre anni e con la multa da euro 31 a euro 516,50". Il suddetto art. 91 stabilisce che le cose aventi rilievo culturale indicate all'art. 10 del codice stesso, "da chiunque e in qualunque modo ritrovate nel sottosuolo o sui fondali marini appartengono allo Stato e, a seconda che siano immobili o mobili, fanno parte del demanio o del patrimonio indisponibile, ai sensi degli articoli 822 e 826 del codice civile". I soggetti attivi del delitto di cui all'art. 176 del Codice possono essere sia l'autore di ricerche autorizzate ai sensi dell'art. 89 del Codice che il ricercatore abusivo o anche coloro che realizzano una scoperta occasionale. Le fattispecie appena esaminate, quasi tutte delineate nella forma della contravvenzione con sanzioni dell'arresto e dell'ammenda piuttosto miti, sembrano non riuscire a tutelare i beni culturali dalle condotte che, nella realtà, risultano più aggressive e lesive, manca, infatti, la punizione di diverse condotte rilevanti che, attualmente, sono state inserite nel disegno di legge AC 4220. Inoltre, se pur formulate nella forma di reato comune, spesso appaiono, nei fatti, reati propri, circoscrivendo il raggio di tutela e di azione della tutela penale.

Per quanto riguarda, invece, il reato di „Danneggiamento al patrimonio archeologico, storico o artistico nazionale“, previsto dall'art. 733 c.p., nel corso del tempo tale norma ha mostrato evidenti limiti strutturali e operativi, tanto da essere ormai ritenuta inoperante ed inapplicabile. La formulazione della norma risente, purtroppo, di alcune caratteristiche tipiche dell'epoca, il 1930, in cui è stata inserita nel codice penale. La norma è travolta dall'enfasi propagandistica 
dell'epoca, piuttosto che da una funzionale operatività in merito alla tutela dei beni culturali. Si tratta di una contravvenzione, inserita nel libro III, titolo II, rubricato delle contravvenzioni concernenti l'attività sociale della pubblica amministrazione. Pur essendo stato formulato come reato comune, nell'esplicazione dell'oggetto tutelato, è sembrato tuttavia essere un reato proprio. Di fatto, è il solo proprietario che può distruggere, deteriorare o danneggiare. L'art. 733 c.p. stabilisce, infatti, che: „Chiunque distrugge, deteriora o comunque danneggia un monumento o una altra cosa propria di cui gli sia noto il rilevante pregio, è punito, se dal fatto deriva un nocumento al patrimonio archeologico, storico o artistico nazionale, con l'arresto fino ad un anno o con l'ammenda non inferiore a euro 2.065,00. Può essere ordinata la confisca della cosa deteriorata o comunque danneggiata". Si tratta, però, di un limite ampiamente superato nella giurisprudenza più recente, la quale ha evidenziato che il soggetto attivo possa essere il proprietario o chiunque stia godendo della disponibilità del bene, sia essa diffusa o collettiva ${ }^{6}$.

Un altro limite applicativo discende però dall'inciso „gli sia noto il rilevante pregio" che rende l'indagine dell'elemento psicologico, nella pratica, piuttosto difficile o quantomeno non sempre pacifico.

Inoltre, dalla condotta tipizzata deve derivare un nocumento al patrimonio archeologico, storico e artistico nazionale: si tratta di un reato di evento, ma di un evento di dimensioni ampie. Da questa precisazione deriva, forse, il vero limite operativo della norma affetta da evidente gigantismo ${ }^{7}$. Dalla distruzione, deterioramento o comunque danneggiamento di un monumento o di altra cosa deve derivare un nocumento non al monumento o alla cosa danneggiata, ma al patrimonio archeologico, storico e artistico nazionale. Vi è una strana asimmetria tra il bene, come oggetto tutelato, e l'oggetto del nocumento, coincidente con il patrimonio nazionale, che rende la tutela apprestata dall'art. 733 c.p. limitativa, poco pragmatica e difficilmente applicabile.

\section{IL RECENTE DISEGNO DI LEGGE AC 4220: L'ASSORBIMENTO NEL CODICE PENALE DELLE FATTISPECIE PENALI A TUTELA DEL PATRIMONIO CULTURALE}

Nonostante la legislazione italiana, rispetto alle legislazioni di altri Paesi, in tema di tutela penale dei beni culturali sia da sempre ritenuta severa, nell'applicazione pratica e in un bilancio applicativo ex post della stessa sembra, invece, non essere completa ed efficace ${ }^{8}$. La previsione sanzionatoria

6 Cass. pen. Sez. III, 24 ottobre 2008, n. 42893. Cass. Pen. 8 luglio 2009, n. 3057.

7 Sul punto, GATTA G.L., Abolitio criminis e successione di norme integratrici: teoria e prassi, Giuffrè, Milano, 2008, p. 293.

8 Per un approfondimento cfr. TRONCONE P., La tutela penale del patrimonio culturale italiano e il deterioramento strutturale del reato dell'art. 733 c.p., in Diritto Penale Contemporaneo, aprile 2016. 
appare eccessivamente mite, e, pertanto, non efficace, in chiave deterrente, a disincentivare la commissione di condotte attive o omissive lesive del patrimonio culturale, artistico ed archeologico.

La tutela penale dei beni culturali inserita come legislazione speciale nel Codice dei Beni Culturali e del Paesaggio non ha trovato un'applicazione concreta, restando, invece, assicurata da altre norme, come la ricettazione o il furto, che sono presenti nel codice penale, ma che non sono armonizzate con la materia di cui trattasi, nè con il bene che si intende proteggere ${ }^{9}$. Altresì le norme attualmente previste dal Codice Penale non riescono a garantire la tutela auspicata ${ }^{10}$. Per punire il furto di beni culturali, attualmente, si può solo, e non sempre, ricorrere alla circostanza aggravante del furto prevista nell'art. $625, \mathrm{n} .7$; in tema di danneggiamento sono presenti i medesimi inconvenienti, di impostazione e di tecnica, nonostante un intervento legislativo che almeno cita espressamente il patrimonio storico artistico come oggetto dell'aggravante dell'art. 635 c.p., comma 2 , n. 3. Alla precisazione dell'oggetto materiale non si è accompagnato un intervento sugli spazi vuoti di tutela, cioè le ipotesi di danneggiamento colposo (frequentissime e non coperte dalla disposizione dell'art. 635 c.p., come anche da quella dell'art. 639 c.p., che concernono fattispecie dolose) e di danneggiamento su cosa propria. A coprire queste lacune dovrebbe contribuire la contravvenzione dell'art. 733 c.p., rubricata: "Danneggiamento al patrimonio archeologico, storico o artistico nazionale”, unica norma espressamente destinata alla tutela dei beni culturali nel nostro codice e che però, dati i suoi limiti strutturali, ha avuto, in giurisprudenza, un'applicazione contrastata e marginale ed è stata definita da Ferrando Mantovani „una contravvenzione gigante, di rara verificazione pratica ${ }^{11}$ “.

9 Per una riflessione generale sulle figure di reato del codice penale, applicabili per garantire una tutela penale dei beni culturali, si rinvia a PECORELLA G., Furto comune, in Enc. del dir., XVIII, Giuffrè, Milano, 1969, pag. 329. Sulla specifica questione del furto aggravato e sulla sostenibilità di una efficacia autonoma dell'incriminazione importante è la riflessione di DEMURO G.P., Un proposta di riforma dei reati contro $i$ beni culturali, in Riv.it.dir. e proc. pen., 2002, pag. 1358: "Sotto un profilo di tecnica giuridica, l'applicazione della circostanza può essere completamente elisa dal concorso di circostanze attenuanti ritenute dal giudice prevalenti nel giudizio di bilanciamento".

10 Per un approfondimento, CARAMAZZA I. F., In tema di repressione penale dei danneggiamenti recati al patrimonio storico, artistico ed archeologico nazionale, in Rass. Avv. Stato, 1966, 1, pag. 744. DI GIOVINE G., Appunti sulla tutela degli immobili di interesse artistico o storico, in Riv. giur. edil., 1971, pag. 697. BELLACOSA M., Patrimonio archeologico, storico e artistico nazionale (tutela penale del), in Enc. giur. Treccani, XXII, Roma, 1990, pag. 1. CARRA D., Danneggiamento al patrimonio archeologico, storico o artistico nazionale, in Trattato di diritto penale - Parte speciale. Vol. XI Le contravvenzioni, a cura di Cadoppi A., Canestrari S., Papa M., Manna A., UTET, Torino, 2012, pag. 558. LA GRECA G., Il danneggiamento al patrimonio archeologico, storico e artistico nazionale (commento), in Giurisprudenza sistematica di diritto penale, diretta da F. Bricola e V. Zagrebelsky, UTET, Torino, 1996, pag. 525.

11 MANTOVANI F., Lineamenti della tutela del patrimonio artistico, in Riv. it. dir. e proc. pen., 1976, pag. 77. Nello stesso senso, MANES V., La tutela penale, in Il diritto dei beni culturali, a cura di Barbati, Cammelli, Sciullo, Bologna, 2006, pag. 262. 
Da questo punto di vista, la tutela del paessaggio e del patrimonio storico e artistico della Nazione" così come auspicata dalla Costituzione non pare, da un punto di vista penale, poter essere effettiva, né sotto il profilo della struttura del reato né sotto il profilo sanzionatorio.

Così come è indubbiamente evidente l'eccessiva importanza relegata all'anticipazione della tutela penale attraverso l'utilizzo della tecnica del pericolo astratto. Il nostro appare, pertanto, un ordinamento severo quando non solo non c'è lesione del bene, ma anche quando la previsione della pericolosità è solo astratta, mentre tale impostazione severa si perde quando il tipo di offesa è quello della lesione.

$\mathrm{E}^{6}$, pertanto, indubbia la necessità di una protezione diretta dei beni culturali, inserita nel Codice Penale, con una previsione sanzionatoria più decisa ma, soprattutto, con una formulazione della struttura dei reati realmente protettiva, chiara ed efficace.

Il recente disegno di legge appare, quindi, andare in questa direzione ed essere, pertanto, quanto mai opportuno.

In primo luogo, perchè modifica direttamente il Codice penale, inserendo il nuovo titolo VIII bis, rubricato „Dei delitti contro il patrimonio culturale“ e composto da 6 articoli che introducono nuovi delitti a tutela del patrimonio culturale, innalzando le pene esistenti e introducendo anche aggravanti se i reati comuni sono commessi contro beni culturali.

I nuovi reati introdotti, in particolare, sono: il furto di beni culturali, l'appropriazione indebita di beni culturali, la ricettazione di beni culturali, il riciclaggio di beni culturali, l'illecita detenzione di beni culturali, le violazioni in materia di alienazione dei beni culturali, il danneggiamento, il deturpamento, l'imbrattamento e l'uso illecito di beni culturali o paesaggistici, il danneggiamento, il deturpamento e imbrattamento colposi di beni culturali o paesaggistici.

L'art. 518-bis punisce il furto di beni culturali e prevede che: „E punito con la reclusione da due a otto anni chiunque si impossessa di un bene culturale, sottraendolo a chi lo detiene, al fine di trarne profitto per sé o per altri. La pena è della reclusione da quattro a dodici anni se il reato è aggravato da una o più delle circostanze previste nel primo comma dell'articolo 625 o se il furto di beni culturali appartenenti allo Stato è commesso da chi abbia ottenuto la concessione di ricerca prevista dalla legge". Si tratta, finalmente, di una norma ad hoc.

L'art. 518-ter punisce l'appropriazione indebita di beni culturali e prevede che: „Chiunque, per procurare a sé o ad altri un ingiusto profitto, si appropria di un bene culturale altrui di cui abbia, a qualsiasi titolo, il possesso è punito con la reclusione da uno a quattro anni. Se il fatto è commesso su cose possedute a titolo di deposito necessario, la pena è au-mentata". Anche qui la struttura della norma segue quella dell'appropriazione indebita ex art. 646 c.p. Ma l'oggetto e il bene tutelato riguardano i beni culturali. 
L'art. 518-quater punisce la ricettazione di beni culturali prevedendo che: „Fuori dei casi di concorso nel reato, chi, al fine di procurare a sé o ad altri un profitto, acquista, riceve od occulta beni culturali provenienti da un qualsiasi delitto, o comunque si intromette nel farli acquistare, ricevere od occultare, $\grave{e}$ punito con la reclusione da tre a dodici anni. Le disposizioni del presente articolo si applicano anche quando l'autore del delitto da cui i beni culturali provengono non è im-putabile o non è punibile, ovvero quando manca una condizione di procedibilità riferita a tale delitto".

L'art. 518-quinquies prevede il reato di riciclaggio di beni culturali sancendo che: „Fuori dei casi di concorso nel reato, chiunque sostituisce o trasferisce beni culturali provenienti da delitto non colposo, ovvero compie in relazione ad essi altre operazioni, in modo da ostacolare l'iden-tificazione della loro provenienza delittuosa, è punito con la reclusione da cinque a quat-tordici anni. La pena è aumentata quando il fatto è commesso nell'esercizio di un'attività professionale. Si applica il secondo comma dell'articolo 518-quater".

L'art. 518-sexies punisce l'illecita detenzione di beni culturali e prevede che: „Fuori dei casi di ricettazione, chiunque detiene un bene culturale sapendo della sua provenienza illecita è punito con la reclusione da sei mesi a cinque anni e con la multa fino a euro 20.000". Questa è una norma di nuova introduzione che colma la grande lacuna della legislazione precedente.

L'art. 518-septies punisce le violazioni in materia di alienazione di beni culturali prevedendo che: „E punito con la reclusione fino a due anni e con la multa fino a euro 80.000: a) chiunque, senza la prescritta autoriz-zazione, aliena beni culturali; b) chiunque, essendovi tenuto, non pre-senta, nel termine di trenta giorni, la denuncia degli atti di trasferimento della proprietà o della detenzione di beni culturali; c) l'alienante di un bene culturale soggetto a prelazione che effettua la consegna della cosa in pendenza del termine di sessanta giorni dalla data di ricezione della de-nuncia di trasferimento".

L'art. 518-octies punisce l'uscita o esportazione illecite di beni culturali e statuisce che: „Chiunque trasferisce all'estero beni culturali, senza attestato di libera circolazione o licenza di esportazione, è punito con la reclusione da uno a quattro anni o con la multa da euro 258 a euro 5.165. La pena prevista al primo comma si applica, altresi, nei confronti di chiunque non fa rientrare nel territorio nazionale, alla scadenza del termine, beni culturali per $i$ quali siano state autorizzate l'uscita o l'esportazione temporanee, nonché nei confronti di chiunque rende dichiarazioni mendaci al fine di comprovare al competente ufficio di esportazione, ai sensi di legge, la non assoggettabilità di cose di interesse culturale ad autorizzazione all'uscita dal territorio nazionale. Se il fatto ̀̀ commesso da chi esercita at-tività di vendita al pubblico o di esposizione a fine di commercio di beni culturali, alla sentenza di condanna consegue l'interdizione ai sensi dell'articolo 30 “. 
L'art. 518 novies prevede il delitto di danneggiamento, deturpamento, imbrattamento e uso illecito di beni culturali o paesaggistici e statuisce che: „Chiunque distrugge, disperde, deteriora o rende, in tutto o in parte, non fruibili beni culturali o paesaggistici è punito con la reclusione da uno a cinque anni. Chiunque, fuori dei casi di cui al primo comma, deturpa o imbratta beni culturali o paesaggistici, ovvero destina beni culturali a un uso incompatibile con il loro carattere storico o artistico ovvero pregiudizievole per la loro conservazione o integrità, è punito con la reclusione da sei mesi a tre anni. La sospensione condizionale della pena è subordinata all'eliminazione delle conseguenze dannose o pericolose del reato ovvero, se il condannato non si oppone, alla prestazione di attività non retribuita a favore della collettività per un tempo determinato, comunque non superiore alla durata della pena sospesa, secondo le modalità indicate dal giudice nella sentenza di condanna".

L'art. 518-decies punisce il danneggiamento, deturpamento e imbrattamento colposi di beni culturali o paesaggistici e prevede che: „Chiunque, co una o più azioni, commette per colpa taluno dei fatti di cui all'articolo 518 novies è punito con la reclusione fino a due anni“. Anche questa è una nuova norma che va a colmare un vuoto di tutela nei confronti delle condotte colpose di danneggiamento, deturpamento e imbrattamento non previste dalla legislazione attuale e, pertanto, non perseguibili e punibili.

L'art. 518-undecies punisce la devastazione e saccheggio di beni culturali prevedendo che: „Chiunque, fuori dei casi preveduti dall'articolo 285, commette fatti di devastazione o di saccheggio aventi ad oggetto beni culturali ovvero istituti e luoghi della cultura è punito con la reclusione da dieci a diciotto anni".

Si tratta di reati comuni che superano i limiti di tutela dell'attuale legislazione, delineati perlopiù come delitti, con pene consistenti anche in termini di reclusione, con condotte che ricalcano i classici reati corrispondenti nelle modalità di condotta previsti dal codice in altri titoli, ma che hanno una struttura più specifica, in primo luogo perchè riguardante direttamente i beni culturali $\mathrm{e}$, poi, perchè formulati con una tecnica di scrittura efficace che supera le attuali fragilità sul tema.

Gli elementi positivi del disegno di legge sono vari e superano di gran lunga i pochi difetti che presenta l'articolato della proposta in questione.

In primo luogo, è assolutamente positiva l'introduzione di un titolo appositamente dedicato ai reati contro il patrimonio culturale, senza l'aggiunta di commi e articoli nei reati già esistenti in altri titoli del codice penale. Ciò dà corpo ed omogeneità alla tutela elevando il patrimonio culturale a nuovo ed autonomo bene giuridico, da considerare di rango primario nell'assetto di valori incarnato nel codice penale. Del resto, la Carta Costituzionale dedica l'art. 9 proprio alla tutela del paesaggio e del patrimonio artistico e storico della Nazione, 
considerandola una principio (la tutela e/o il patrimonio) di rango costituzionale ${ }^{12}$. Inoltre, tale nuovo assetto nella collocazione die reati permette di superare la tendeziale frammentazione e disomogenietà del quadro normativo vigente, così come è considerato da larga parte della dottrina ${ }^{13}$. In secondo luogo, la creazione di nuove fattispecie di reato prima non esistenti, tra i quali, per esempio, il furto, la ricettazione o l'illecita detenzione, ha permesso di superare, quantomeno in una prospettiva de iure condendo, la debole tutela prevista dalla legslazione attuale che contiene illeciti, perlopiù di natura contravvenzionale, di danno o di pericolo concreto che proteggono la disciplina amministrativa di tutela dei beni culturali piuttosto che i beni culturali in sè. Nel disegno di legge, questa tiepida propensione verso la tutela della disciplina amministrativa dei beni culturali viene messa da parte, per far spazio a fattispecie di tipo delittuoso che individuano condotte lesive direttamente dei beni culturali. In questo senso vanno anche le sanzioni previste nettamente più incisive e severe, anche grazie alla strutturazione delle fattispecie nella forma di delitti e non di contravvenzioni. Ma non si tratta solo di semplice inasprimento dell'apparato sanzionatorio, quanto, piuttosto, di un delineare con chiarezza e pervasività le fattispecie incriminatrici.

Inoltre, è da considerare positivo l'assorbimento in tale titolo di reati già presenti nel Codice dei Beni Culturali e del Paesaggio, quali la vendita di opere $d$ 'arte senza autorizzazione o in pendenza di prelazione, l'esportazione illecita il danneggiamento o l'imbrattamento e la contraffazione, con una migliore tecnica di formulazione della fattispecie, più incisiva, determinata e chiara e con una omogeneità di tutela complessiva che attualmente non segue più la divisione tra reati del codice penale e reati del codice dei beni e del paesaggio.

Anche la previsione, del tutto innovativa, della punizione del traffico illecito da parte di attività organizzate con l'introduzione dell'art. 518 quaterdecies il quale prevede che: „Chiunque, al fine di conseguire un ingiusto profitto o vantaggio, con più operazioni e attraverso l'allestimento di mezzi e attività continuative organizzate, trasferisce, aliena, scava clandestinamente o comunque gestisce illecitamente beni culturali è punito con la reclusione da due a otto anni“", costituisce un rilevante passo in avanti nella protezione del patrimonio culturale, che tiene conto del riscontro pratico della rilevanza nell'economia illegale del

12 Per un approfondimento cfr. MONTANARI T., Art. 9. Costituzione italiana, Carocci ed., Roma, nel quale si legge a p. 49, „La Repubblica tutela. Di fronte all'oscillazione che segna il dibattito alla Costituente (dove si alternano, si è visto, i concetti di vigilanza e protezione, Tristano Codignola affermò perentorio il 20 aprile 1947: „Lo Stato non protegge, tutela“. Con ogni probabilità Codignola aveva nelle orecchi e nella mente le parole della legge 1089/1939, intitolata proprio alla Tutela delle cose di interesse artistico e storico. L'Assemblea fece suo questo punto di vista e fu un bene, perché se il concetto di vigilanza appare decisamente passivo quello di protezione ha in sé qualcosa di inevitabilmente positivo...".

13 Si veda fra tutti, MANACORDA S., La circolazione illecita dei beni culturali nella prospettiva penalistica: problemi e prospettive di riforma, in AA. VV., Circolazione dei beni culturali mobili e tutela penale: un'analisi di diritto interno, comparato ed internazionale, Milano, 2015, p. 6. 
mercato nero dell'arte da parte delle organizzazioni criminali.

Un altro aspetto positivo riguarda l'obbligo di confisca dei beni culturali, anche per equivalente.

$\mathrm{Vi}$ è, inoltre, la previsione dell'applicazione di tali disposizioni anche quando il fatto è commesso all'estero in danno del patrimonio culturale nazionale.

In linea con quanto previsto dalla Convenzione di Nicosia all'art. 13, viene prevista l'estensione della responsabilità degli enti ex d.lgs. 231/2001 anche ai nuovi delitti contro il patrimonio culturale con sanzioni pecuniarie ad hoc calcolare in quote a seconda del reato posto in essere;

Manca, probabilmente, una definizione di beni culturali che renda agevole l'interpretazione per evitare di delegare scelte politico criminali al singolo giudice e per non incorrere in una distinzione non chiara tra beni pubblici e beni privati, ma nel complesso di tratta di una riforma ben fatta e più che opportuna ${ }^{14}$.

\section{QUALCHE OSSERVAZIONE CONCLUSIVA}

I beni culturali di interesse artistico e archeologico permettono di trasmettere al pubblico, nel corso del tempo, il patrimonio culturale che si forma nei vari periodi storici e consentono di comunicare la storia, la tecnica, la creatività, la conoscenza delle varie fasi dello sviluppo del patrimonio culturale. Contengono un enorme interesse pubblico e costituiscono il seme con il quale, permettendo a chiunque di poterli contemplare e conoscere, si trasmette la bellezza e la conoscenza da un periodo storico ad un altro, da una fase ad un'altra, da un movimento ad un altro, nel corso della storia. $E^{‘}$ per questo che la nostra Costituzione ha inserito la tutela dei beni culturali nei principi fondamentali.

In questa luce, il nuovo disegno di legge si armonizza all'importanza di questo principio fondamentale, una rosa nel seme che sboccia nel succedersi nel tempo e che necessita di essere custodita e protetta per garantirne la diffusione a tutti coloro i quali desiderano conoscerli.

\section{BIBLIOGRAFIA ESSENZIALE}

BELLACOSA M., Patrimonio archeologico, storico e artistico nazionale (tutela penale del), in Enc. giur. Treccani, XXII, Roma, 1990.

BENINI S., Circa il danneggiamento di beni culturali, in Foro it., 1999, II.

14 Per una disamina più accurata dei punti critici della riforma si rinvia a L. D'AGOSTINO, Dalla "vittoria di Nicosia" alla "navetta" legislativa. I nuovi orizzonti normativ nel contrasto ai traffici illeciti di beni culturali, in Diritto penale Contemporaneo, Milano, n. 1/2018. Tali profili di criticità sono emersi nel corso delle audizioni in Commissione Giustizia della Camera dei Deputati del 2 maggio 2017 in ordine alla definizione del bene culturale, in ordine alla fattispecie di nuova introduzione di detenzione di bene culturale che punisce una generica situazione possessoria sapendo della sua provenienza illecita senza però aver contribuito al ritrovamento, che rappresenta una scelta di eccessiva criminalizzazione e, infine, in ordine alla mancata punibilitàdi condotte di import illecito. Il resoconto delle audizioni è disponibile sul sito della Camera nella sezione dedicata al disegno di legge AC-4220. 
CANTUCCI M., La tutela giuridica delle cose di interesse artistico o storico, Padova, Cedam, 1953.

CARAMAZZA I. F., In tema di repressione penale dei danneggiamenti recati al patrimonio storico, artistico ed archeologico nazionale, in Rass. Avv. Stato, 1966.

CARRA D., Danneggiamento al patrimonio archeologico, storico o artistico nazionale, in Trattato di diritto penale - Parte speciale. Vol. XI Le contravvenzioni, a cura di Cadoppi A., Canestrari S., Papa M., Manna A., UTET, Torino, 2012.

DEMURO G.P., Verso la riforma dei reati contro $i$ beni culturali: approvato dal Consiglio dei ministri un disegno di legge delega per l'introduzione nel codice penale delle più gravi forme di aggressione al patrimonio culturale, in Rivista di Diritto penale contemporaneo, fasc. 1/2017.

DEMURO G.P., Un proposta di riforma dei reati contro $i$ beni culturali, in Riv. it.dir. e proc.pen., 2002.

DEMURO G.P., Beni culturali e tecniche di tutela penale, Giuffrè, Milano, 2002.

DI GIOVINE G., Appunti sulla tutela degli immobili di interesse artistico o storico, in Riv. giur. Edil., 1971.

GIANNINI M.S., I beni culturali, in Riv. trim. di dir. Pubb., 1976.

LA GRECA G., Il danneggiamento al patrimonio archeologico, storico e artistico nazionale (commento), in Giurisprudenza sistematica di diritto penale, diretta da F. Bricola e V. Zagrebelsky, UTET, Torino, 1996.

LAZZARI C., L'art.733 C.P., una norma priva di virtualità applicativa, in Cass. Pen., 2000.

MANES V., La tutela penale, in Il diritto dei beni culturali, a cura di Barbati, Cammelli, Sciullo, Bologna, 2006.

MANTOVANI F., Lineamenti della tutela del patrimonio artistico, in Riv. it. dir. e proc. Pen., 1976.

MOCCIA S., Il diritto penale tra essere e valore. Funzione della pena e sistematica teleologica, Esi, Napoli, 1992.

MORGANTE G., Nuove tecniche repressive nei confronti delle condotte di aggressione al patrimonio culturale, Leg. Pen., 1998.

MUZZICA R., Confisca dei beni culturali e prescrizione: contro o oltre Varvara?, in Rivista diritto penale contemporaneo, 23 novembre 2015.

PATALANO V., Beni costituzionali e tutela penale degli interessi economici, in Studi in onore di Giuliano Vassalli, vol. I, Giuffrè, Milano, 1991. 
PIOLETTI G., Patrimonio artistico e storico nazionale (reati contro il), in Enc. del dir., Giuffrè, Milano, XXXII, 1982.

PECORELlA G., Furto comune, in Enc. del dir., XVIII, Giuffrè, Milano, 1969.

ROLLA G., Beni culturali e funzione sociale, in Le regioni, 1987, fasc. 1-2.

TRONCONE P., La tutela penale del patrimonio culturale italiano e il deterioramento strutturale del reato dell'art. 733 c.p., Rivista diritto penale contemporaneo, $1 / 2016$.

ZANOTTI M., L'art. 733 c.p. e la tutela del patrimonio archeologico, storico o artistico nazionale, in Cass. Pen., 1997. 


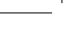

